\title{
Aspirin and other non-steroidal anti- inflammatory drugs and depression, anxiety, and stress-related disorders following a cancer diagnosis: a nationwide register-based cohort study
}

\author{
Kejia Hu' ${ }^{1}$, Arvid Sjölander ${ }^{2}$, Donghao Lu ${ }^{2,3,4}$, Adam K. Walker ${ }^{5,6,7}$, Erica K. Sloan ${ }^{5}$, Katja Fall, ${ }^{1,8}$, \\ Unnur Valdimarsdóttir ${ }^{2,9}$, Per Hall ${ }^{2,10}$, Karin E. Smedby ${ }^{11}$ and Fang Fang ${ }^{1 *}$
}

\begin{abstract}
Background: Cancer patients have a highly increased risk of psychiatric disorders following diagnosis, compared with cancer-free individuals. Inflammation is involved in the development of both cancer and psychiatric disorders. The role of non-steroidal anti-inflammatory drugs (NSAIDs) in the subsequent risk of psychiatric disorders after cancer diagnosis is however unknown.

Methods: We performed a cohort study of all patients diagnosed with a first primary malignancy between July 2006 and December 2013 in Sweden. Cox proportional hazards models were used to assess the association of NSAI $\mathrm{D}$ use during the year before cancer diagnosis with the risk of depression, anxiety, and stress-related disorders during the first year after cancer diagnosis.

Results: Among 316,904 patients identified, 5613 patients received a diagnosis of depression, anxiety, or stressrelated disorders during the year after cancer diagnosis. Compared with no use of NSAIDs, the use of aspirin alone was associated with a lower rate of depression, anxiety, and stress-related disorders (hazard ratio [HR], 0.88; 95\% confidence interval [Cl], 0.81 to 0.97$)$, whereas the use of non-aspirin NSAIDs alone was associated with a higher rate (HR, 1.24; $95 \% \mathrm{Cl}, 1.15$ to 1.32), after adjustment for sociodemographic factors, comorbidity, indications for NSAl $\mathrm{D}$ use, and cancer characteristics. The association of aspirin with reduced rate of depression, anxiety, and stressrelated disorders was strongest for current use ( $\mathrm{HR}, 0.84 ; 95 \% \mathrm{Cl}, 0.75$ to 0.93$)$, low-dose use (HR, 0.88; 95\% Cl, 0.80 to 0.98$)$, long-term use ( $\mathrm{HR}, 0.84 ; 95 \% \mathrm{Cl}, 0.76$ to 0.94$)$, and among patients with cardiovascular disease (HR, 0.81 ; $95 \% \mathrm{Cl}, 0.68$ to 0.95$)$ or breast cancer ( $\mathrm{HR}, 0.74 ; 95 \% \mathrm{Cl}, 0.56$ to 0.98$)$.
\end{abstract}

Conclusion: Pre-diagnostic use of aspirin was associated with a decreased risk of depression, anxiety, and stressrelated disorders during the first year following cancer diagnosis.

Keywords: Aspirin, Anti-inflammatory agents, non-steroidal, Mental disorders, Neoplasms

\footnotetext{
* Correspondence: fang.fang@ki.se

'Unit of Integrative Epidemiology, Institute of Environmental Medicine,

Karolinska Institutet, Box 210, 17177 Stockholm, Sweden

Full list of author information is available at the end of the article
}

C C The Author(s). 2020 Open Access This article is licensed under a Creative Commons Attribution 4.0 International License, which permits use, sharing, adaptation, distribution and reproduction in any medium or format, as long as you give appropriate credit to the original author(s) and the source, provide a link to the Creative Commons licence, and indicate if changes were made. The images or other third party material in this article are included in the article's Creative Commons licence, unless indicated otherwise in a credit line to the material. If material is not included in the article's Creative Commons licence and your intended use is not permitted by statutory regulation or exceeds the permitted use, you will need to obtain permission directly from the copyright holder. To view a copy of this licence, visit http://creativecommons.org/licenses/by/4.0/. The Creative Commons Public Domain Dedication waiver (http://creativecommons.org/publicdomain/zero/1.0/) applies to the data made available in this article, unless otherwise stated in a credit line to the data. 


\section{Background}

Psychiatric disorders are common comorbidities among patients with cancer [1] and may contribute to increased morbidity [2] and mortality [3, 4] after cancer diagnosis. In a previous study, we reported a highly increased risk of common psychiatric disorders, including depression, anxiety, and stress-related disorders, namely posttraumatic stress disorder (PTSD), acute stress reaction, adjustment disorder, and other stress reactions, among cancer patients, especially during the first year after cancer diagnosis [5]. Underlying reasons for such increased risk may include a severe stress response after receiving cancer diagnosis [6-8] and psychiatric symptoms caused by cancer treatment $[9,10]$, pain $[11]$, and inflammation $[12,13]$. Inflammation in the tumor micro-environment drives tumor development and progression [14] and cancer patients have a high burden of cancer-induced systemic inflammation [15].

Inflammation has been suggested to be involved in the development of depression [16] and other psychiatric disorders [17]. For instance, inflammatory cytokines have been shown to influence neurocircuitry in the brain through the consequence of neurotransmitter signaling [18], including a cascade of behavioral and immune responses that might lead to depression, anxiety [19], and PTSD [19, 20] among vulnerable individuals. Although the link between inflammation and psychiatric disorders has been less explored among patients with cancer, chronic inflammatory disorders have been reported to be risk factors for depression and anxiety among cancer patients [21].

Non-steroidal anti-inflammatory drugs (NSAIDs) are commonly prescribed for pain and inflammation. The anti-inflammatory actions of NSAIDs include inhibition of cyclooxygenase (COX) activity and prostaglandin synthesis [22]. Due to its non-competitive and irreversible acetylation of COX, aspirin is different from non-aspirin NSAIDs in terms of indications and adverse effects [23]. A recent meta-analysis of 26 relatively small randomized clinical trials suggested that NSAIDs play an antidepressant role in patients with major depressive disorder and are reasonably safe [24]. Preclinical studies indicate that the use of aspirin is associated with a lower risk of depression in the general population [25], and among patients with stroke [26] or osteoarthritis [27]. There is, however, a lack of evidence in this regard among cancer patients and on psychiatric disorders other than depression. Our recent preclinical study showed that low-dose aspirin might counteract the inflammation-related cognitive impairment in a mouse model of breast cancer [28]. It is therefore plausible that aspirin may help to prevent inflammation-related psychiatric disorders among cancer patients.

To this end, we performed a nationwide register-based study in Sweden to investigate the role of pre-diagnostic use of NSAIDs, especially aspirin, in the risk of depression, anxiety, and stress-related disorders following cancer diagnosis. Our hypothesis was that pre-diagnostic use of NSAIDs, especially aspirin, is associated with a decreased risk of depression, anxiety, and stress-related disorders following cancer diagnosis, compared with no use of NSAIDs.

\section{Methods}

Study design

From the Swedish Cancer Register [29], we identified 338,009 patients that were diagnosed with a first primary malignancy between July 1, 2006, and December 31, 2013. Through cross-linkages with the Swedish Causes of Death Register, Migration Register, and Patient Register, we followed these patients individually from the date of cancer diagnosis until death, emigration from Sweden, or 1 year after cancer diagnosis, whichever came first, using the Swedish personal identity numbers. We focused on the first year after cancer diagnosis because the risk of psychiatric disorders appears to be highest immediately after cancer diagnosis [5]. Patients were excluded from the analysis if they had conflicting information (i.e., died or emigrated before cancer diagnosis) $(n=982)$, or were diagnosed at autopsy $(n=2336)$. Because we aimed at assessing the risk of newly diagnosed psychiatric disorders, patients with preexisting depression, anxiety, or stress-related disorder before cancer diagnosis ascertained since 1973 onward according to the Patient Register [30] were also excluded ( $n=17,787)$, leaving 316,904 patients in the final analysis (Fig. 1). We hypothesized the newly onset depression, anxiety, and stress-related disorders after cancer diagnosis might be more closely related to cancer-related inflammation and the psychological stress patients experienced after receiving a cancer diagnosis.

\section{Pre-diagnostic use of NSAIDs}

We linked the cohort of cancer patients to the Swedish Prescribed Drug Register, which contains information on all prescribed medications that are dispensed in Sweden since July 2005 [31]. The vast majority of prescribed medications are subsidized with a celling of co-payment in Sweden. Medications are coded according to the Anatomical Therapeutic Chemical (ATC) Classification System in this register. We identified all records of NSAIDs (aspirin: N02BA01, N02BA51, B01AC06; non-aspirin NSAIDs: M01A) dispensed within 365 days before cancer diagnosis. Patients were then classified into the mutually exclusive categories "no use of NSAIDs," "use of aspirin alone," "use of non-aspirin NSAIDs alone," or "use of both." We also grouped the medications by cyclooxygenase selectivity. Aspirin, flurbiprofen, ketoprofen, fenoprofen, tolmetin, and oxaprozin were defined as COX-1 selective NSAIDs, 


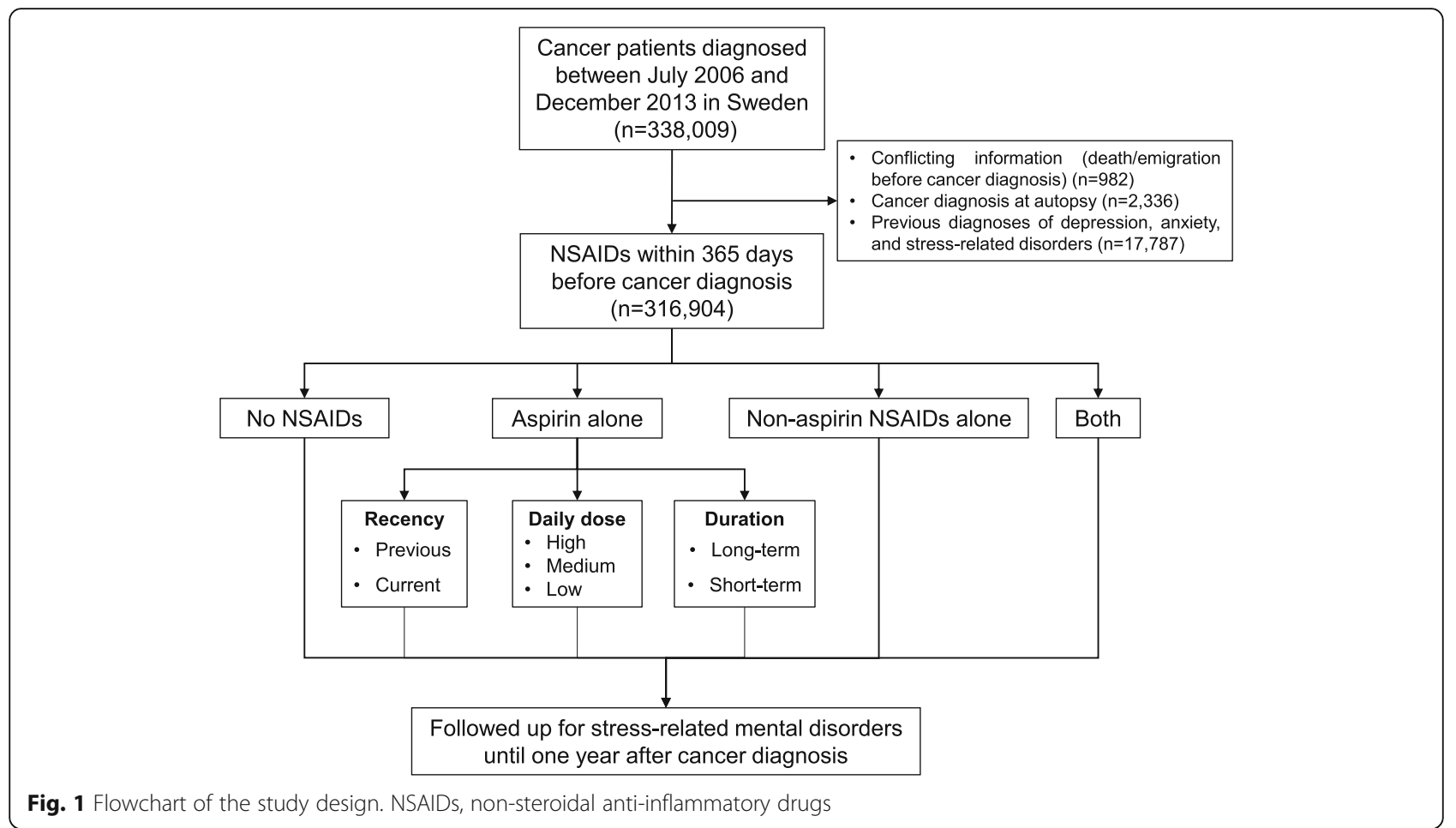

whereas coxibs, meloxicam, etodolac, mefenamic acid, and diclofenac were defined as COX-2 selective NSAIDs. Nonselective NSAIDs and use of both categories were collapsed into an additional group.

As aspirin is the most frequently used NSAID, and our animal study suggested its protective role in cognitive wellbeing in a mouse model of breast cancer [28], we specifically studied aspirin and categorized the use of aspirin according to recency of use, daily dose, and duration of use. Recency of use was defined by the time between cancer diagnosis and last dispensation during 365 days before cancer diagnosis, and classified as "previous use" if the last dispensation was more than 90 days before cancer diagnosis or "current use" if no more than 90 days. The information of daily dose was extracted from the prescription text, and a mean daily dose was calculated from the prescribed daily dose of each dispensation. The daily dose was then categorized as low (20-150 $\mathrm{mg}$ ), medium (151-300 mg), or high (> $300 \mathrm{mg}$ ). Duration of use was defined as the number of days that patients were supplied with aspirin, as estimated by the total dispensed dose divided by the mean daily dose. We defined a patient as a long-term user if the estimated duration was 300 days or more (the median of all duration values). Possible regimens of aspirin use, combining information on recency, dose, and duration, were also examined.

\section{Post-diagnostic depression, anxiety, and stress-related disorders}

Through the Patient Register, we identified all patients with an inpatient or outpatient hospital visit that resulted in a diagnosis of psychiatric disorders [International Statistical Classification of Diseases and Related Health Problems 10th Revision (ICD-10): F10-F99] during the follow-up (from date of cancer diagnosis until up to 1 year after cancer diagnosis). We then limited the analysis to three groups of psychiatric disorders that are common among cancer patients $[32,33]$ and potentially related to inflammation [34], including (1) depression disorders (ICD-10: F32, F33), (2) anxiety disorders (ICD10: F40, F41), and (3) stress-related disorders, namely PTSD, acute stress reaction, adjustment disorder, and other stress reactions (ICD-10: F43).

\section{Covariables}

Information on age at diagnosis, date of diagnosis, cancer type, and cancer stage was obtained from the Cancer Register. We studied the most common cancer types, including prostate cancer, breast cancer, gastrointestinal cancers, lung cancer, skin cancer, kidney and bladder cancers, gynecological cancers, hematological malignancies, and other less common cancers including tumor of the central nervous system. Using the European Network of Cancer Registries Condensed TNM Scheme and International Federation of Gynecology and Obstetrics (FIGO) staging system, we classified non-hematological malignancies as localized limited (T-localized/N0/M0 or FIGO 0-I), localized advanced (T-advanced/N0/M0 or FIGO II), regional spread (any $\mathrm{T} / \mathrm{N}+/ \mathrm{M} 0$ or FIGO III), distant metastasis (any T/any N/M+ or FIGO IV), and unknown stage. "Mx" was regarded as "MO" because 
"Mx" is commonly used when there are no clinical indications of distant metastasis.

We calculated chronic disease score based on the medications used during the year before cancer diagnosis, as a comorbidity measure $[35,36]$. We excluded psychiatric medications, anti-inflammatory drugs, and analgesics from this calculation because they are related to the outcome and exposure of interest in the present study. We also ascertained potential indications for NSAID use through the Patient Register and classified them as cardiovascular diseases (ICD-10: G45.9, I00-02, I05-09, I20-21, I30, I32-33, I38-40, I63, I65.2, I80-83, I88, Z95), inflammatory musculoskeletal conditions (ICD-10: M05-19, M45-46, M60, M65), inflammatory systemic diseases (ICD-10: M30-36), and pain and fever (ICD-10: F45.4, G43-44, G50.0-50.1, M25.5, M54.5, M54.9, M79.1, N94.4, R50.8-50.9, R51-52). We further linked the cohort to the Longitudinal Integrated Database for Health Insurance and Labour Market Studies (LISA) [37] for ascertainment of various potential confounders, including educational level, occupation, region of residence (east, south, and north according to the first-level Classification of Territorial Units for Statistics), and marital status at cancer diagnosis.

\section{Statistical analysis}

We described baseline characteristics of the cancer patients according to their levels of NSAID use. We calculated the incidence rates (IRs) of depression, anxiety, and stress-related disorders by diving the number of patients that received a diagnosis of such disorders by the accumulated number of person-years during follow-up. We then investigated the rate of the studied disorders in relation to the different levels of NSAID use using Cox proportional hazards models. In the Cox models, the "time to event" data were used by setting the date of cancer diagnosis (from the Cancer Register) as the time when an individual started being at risk for the outcome, the date of diagnosis of depression, anxiety, or stressrelated disorder (from Patient Register) as the time of event, and the earliest date among death (from the Causes of Death Register), emigration (from the Migration Register), and 1 year after cancer diagnosis as the time of censoring if no event happened. We adjusted for age at cancer diagnosis, sex, calendar year of cancer diagnosis, educational level, occupation, place of residence, marital status, and comorbidity in model 1 and additionally adjusted for indications for NSAID use in model 2. We created one dummy variable per indication of NSAID use. In model 3, we further adjusted for cancer type and cancer stage. We first analyzed all studied disorders together and then analyzed separately depression, anxiety, and stress-related disorders. We then focused on aspirin and studied the effect of recency of use, daily dose, duration of use, and combined regimens of aspirin use. To calculate stratum-specific HRs, we fitted separate models for each level of sociodemographic factors, comorbidity, indications for use, and cancer characteristics for aspirin use as well as for current, low-dose, and long-term aspirin use. To test for effect modification by these variables, we included interaction terms between NSAID use and these variables in the multivariable models and used Wald tests to test the statistical significance of the estimated interaction terms (for one exposure and one stratification variable at a time).

Because patients with other psychiatric disorders might be more likely to use NSAIDs and at higher risk of studied disorders after cancer diagnosis, we performed a sensitivity analysis where cancer patients with any preexisting psychiatric disorders before cancer diagnosis were excluded. Additionally, as patients with gastrointestinal symptoms might avoid the use of NSAIDs and might have a higher risk of depression, anxiety, and stress-related disorders in general, we conducted another sensitivity analysis where we stratified the analysis by use of proton pump inhibitors, defined through at least two dispensations, within 1 year before cancer diagnosis. In all analyses, time since cancer diagnosis was used as the underlying timescale. We used Schoenfeld residuals to test the proportional hazards assumption for the main exposure (i.e., NSAID use) in all models and found no major deviation from the assumption.

The statistical analyses were performed using SAS, version 9.4, SAS Institute and Stata, version 16, StataCorp LP. We used a two-sided $P<0.05$ to indicate statistical significance.

\section{Results}

Among the 316,904 patients included in the analysis, the median age at cancer diagnosis was 68 years and $53.4 \%$ were male. $58,761(18.5 \%)$ of these patients used aspirin alone and 49,059 (15.4\%) used non-aspirin NSAIDs alone during the year before cancer diagnosis (Fig. 1). Cancer patients who used aspirin alone were more likely to be male, older, less educated, were less likely to be working, had more comorbidities, and were more likely to have a deceased partner, compared with patients who did not use any NSAIDs (Table 1). Compared with no users, aspirin users were more likely to have cardiovascular disease, whereas non-aspirin NSAID users were more likely to have inflammatory musculoskeletal conditions such as arthritis.

\section{Types of NSAID use and risk of depression, anxiety, and stress-related disorders}

A total of 5613 patients received a diagnosis of depression, anxiety, and stress-related disorders during the year after cancer diagnosis. Compared with no NSAID use, 
Table 1 Baseline characteristics of cancer patients at the time of cancer diagnosis by exclusive use of NSAIDs

\begin{tabular}{|c|c|c|c|c|}
\hline Characteristics & No NSAIDs & Aspirin & Non-aspirin NSAIDs & Both NSAIDs \\
\hline Number & 194,198 & 58,761 & 49,059 & 14,886 \\
\hline \multicolumn{5}{|l|}{ Year of cancer diagnosis (\%) } \\
\hline $2006-2009$ & $86,249(44.4)$ & $26,731(45.5)$ & $23,287(47.5)$ & $7411(49.8)$ \\
\hline 2010-2013 & $107,949(55.6)$ & $32,030(54.5)$ & $25,772(52.5)$ & $7475(50.2)$ \\
\hline \multicolumn{5}{|l|}{ Sex (\%) } \\
\hline Male & $99,678(51.3)$ & $36,861(62.7)$ & $23,780(48.5)$ & $8910(59.9)$ \\
\hline Female & $94,520(48.7)$ & $21,900(37.3)$ & $25,279(51.5)$ & $5976(40.1)$ \\
\hline Age at cancer diagnosis, mean [SD], years (\%) & $65.7[13.0]$ & $75.4[9.5]$ & $64.8[11.8]$ & $73.3[9.2]$ \\
\hline \multicolumn{5}{|l|}{ Educational level ${ }^{a}(\%)$} \\
\hline Low & $63,369(32.6)$ & $27,762(47.2)$ & $15,859(32.3)$ & $6546(44.0)$ \\
\hline Medium & $76,981(39.6)$ & $20,699(35.2)$ & $20,420(41.6)$ & $5590(37.6)$ \\
\hline High & $51,399(26.5)$ & $9267(15.8)$ & $12,221(24.9)$ & $2513(16.9)$ \\
\hline Unknown & $2449(1.3)$ & $1033(1.8)$ & $559(1.1)$ & $237(1.6)$ \\
\hline \multicolumn{5}{|l|}{ Occupation (\%) } \\
\hline Blue-collar & $28,227(14.5)$ & $2772(4.7)$ & $8472(17.3)$ & $901(6.1)$ \\
\hline White-collar & $46,049(23.7)$ & $4163(7.1)$ & $10,959(22.3)$ & $1215(8.2)$ \\
\hline Not working & $119,190(61.4)$ & $51,743(88.1)$ & $29,460(60.1)$ & $12,742(85.6)$ \\
\hline Unclassified or unknown & $732(0.4)$ & $83(0.1)$ & $168(0.3)$ & $28(0.2)$ \\
\hline \multicolumn{5}{|l|}{ Region of residence ${ }^{\mathrm{b}}(\%)$} \\
\hline East & $71,206(36.7)$ & $19,957(34.0)$ & $17,831(36.3)$ & $4903(32.9)$ \\
\hline South & $87,891(45.3)$ & $26,708(45.5)$ & $22,503(45.9)$ & $6834(45.9)$ \\
\hline North & $35,101(18.1)$ & $12,096(20.6)$ & $8725(17.8)$ & $3149(21.2)$ \\
\hline \multicolumn{5}{|l|}{ Marital status (\%) } \\
\hline Unmarried & $29,383(15.1)$ & $4990(8.5)$ & $6355(13.0)$ & $1101(7.4)$ \\
\hline Married/registered partnership & $108,123(55.7)$ & $31,120(53.0)$ & $28,141(57.4)$ & $8242(55.4)$ \\
\hline Divorced/separated & $30,803(15.9)$ & $8474(14.4)$ & $8734(17.8)$ & $2441(16.4)$ \\
\hline Widow(er)/surviving partner & $25,889(13.3)$ & $14,177(24.1)$ & 5829 (11.9) & $3102(20.8)$ \\
\hline \multicolumn{5}{|l|}{ Chronic disease score ${ }^{c}(\%)$} \\
\hline 0 & $80,567(41.5)$ & $25(0.0)$ & $15,061(30.7)$ & $19(0.1)$ \\
\hline $1-3$ & $95,079(49.0)$ & $25,849(44.0)$ & $29,061(59.2)$ & $5792(38.9)$ \\
\hline$>3$ & $18,552(9.6)$ & $32,887(56.0)$ & $4937(10.1)$ & $9075(61.0)$ \\
\hline \multicolumn{5}{|l|}{ Diagnosis of potential indications for NSAIDs ${ }^{\mathrm{d}}(\%)$} \\
\hline Cardiovascular disease & $30,121(15.5)$ & $28,326(48.2)$ & $7573(15.4)$ & $6823(45.8)$ \\
\hline Inflammatory musculoskeletal condition & $28,002(14.4)$ & $10,981(18.7)$ & $14,905(30.4)$ & $4984(33.5)$ \\
\hline Inflammatory systemic disease & $2792(1.4)$ & $1296(2.2)$ & $1135(2.3)$ & $462(3.1)$ \\
\hline Pain and fever & $2410(1.2)$ & $630(1.1)$ & $1044(2.1)$ & $259(1.7)$ \\
\hline \multicolumn{5}{|l|}{ Cancer stage $^{\mathrm{e}}(\%)$} \\
\hline Localized limited & $57,011(29.4)$ & $14,106(24.0)$ & $13,274(27.1)$ & $3474(23.3)$ \\
\hline Localized advanced & $11,719(6.0)$ & $3906(6.6)$ & $2328(4.7)$ & $743(5.0)$ \\
\hline Regional spread & $20,914(10.8)$ & $5571(9.5)$ & $4730(9.6)$ & $1314(8.8)$ \\
\hline Distant metastasis & $19,155(9.9)$ & $6317(10.8)$ & $5954(12.1)$ & $1934(13.0)$ \\
\hline Unknown & $72,027(37.1)$ & $23,868(40.6)$ & $18,232(37.2)$ & 5929 (39.8) \\
\hline Not applicable $e^{f}$ & $13,372(6.9)$ & $4993(8.5)$ & $4541(9.3)$ & $1492(10.0)$ \\
\hline
\end{tabular}

Cancer type ${ }^{9}(\%)$ 
Table 1 Baseline characteristics of cancer patients at the time of cancer diagnosis by exclusive use of NSAIDs (Continued)

\begin{tabular}{lllll}
\hline Characteristics & No NSAIDs & Aspirin & Non-aspirin NSAIDs & Both NSAIDs \\
\hline Prostate cancer & $37,380(19.2)$ & $12,510(21.3)$ & $9699(19.8)$ & $3309(22.2)$ \\
Breast cancer & $30,135(15.5)$ & $4702(8.0)$ & $7484(15.3)$ & $7313(8.8)$ \\
Gastrointestinal cancer & $34,625(17.8)$ & $11,340(19.3)$ & $7029(14.3)$ & $2381(16.0)$ \\
Lung cancer & $11,126(5.7)$ & $4612(7.8)$ & $3867(7.9)$ & $1426(9.6)$ \\
Non-melanoma skin cancer & $11,619(6.0)$ & $5612(9.6)$ & $2190(4.5)$ & $1057(7.1)$ \\
Melanoma & $11,417(5.9)$ & $2242(3.8)$ & $2233(4.6)$ & $273(5.7)$ \\
Kidney \& bladder cancer & $10,785(5.6)$ & $4764(8.1)$ & $273)$ & $1155(7.8)$ \\
Gynecological cancer & $11,313(5.8)$ & $2438(4.1)$ & $2901(5.9)$ & $615(4.1)$ \\
Hematological malignancy & $13,372(6.9)$ & $4993(8.5)$ & $4541(9.3)$ & $1492(10.0)$ \\
Other cancers & $22,426(11.5)$ & $5548(9.4)$ & $6380(13.0)$ & $1591(10.7)$
\end{tabular}

Cancer patients were categorized into exclusive groups according to their usage of NSAIDs during the year before cancer diagnosis: non-users (never used any NSAID), aspirin users (used aspirin but not non-aspirin), non-aspirin NSAID users (used non-aspirin NSAIDs but not aspirin), users of both NSAIDs (used both aspirin and non-aspirin NSAIDs)

${ }^{a}$ Classified according to years of education: high (college and above), medium (9 years plus 2-3 years secondary school), low (9 years or less), or unknown b Identified through the First-level Classification of Territorial Units for Statistics, NUTS-1

${ }^{c}$ Calculated based on all medications used within 1 year before cancer diagnosis, after excluding psychiatric medications, anti-inflammatory drugs, and analgesics from the original codes list that are directly related to the outcome or exposure of interest

didentified from the Swedish Patient Register since 2001. The four groups of potential indications are non-exclusive

e Defined by European Network of Cancer Registries Condensed TNM Scheme and International Federation of Gynecology and Obstetrics staging system: localized limited (T-localized/NO/MO or FIGO 0-I), localized advanced (T-advanced/NO/MO or FIGO II), regional spread (any T/N+/M0 or FIGO III), distant metastasis (any T/any $\mathrm{N} / \mathrm{M}+$ or FIGO IV), or unknown stage

fHematological malignancies were further divided into five subtypes: leukemia, lymphoma, myeloma, myelodysplastic syndrome, and myeloproliferative neoplasm ${ }^{9}$ Displayed according to the most common cancer types in this population

the use of aspirin alone was associated with a lower rate of the studied disorders in all models (HR, 0.88; 95\% CI, 0.81 to 0.97 in model 3 with adjustment for sociodemographic factors, comorbidity, indications, and cancer characteristics) (Table 2). Similar results were found for depression, anxiety, and stress-related disorders separately (Supplementary Table S1). In contrast, the use of non-aspirin NSAIDs alone was associated with an increased rate of the studied disorders (Table 2 and Supplementary Table S1), compared with no NSAID use. We did not find an association between the use of both aspirin and non-aspirin NSAIDs and the studied disorders. The sensitivity analyses showed similar results after excluding patients with any pre-existing psychiatric disorders (Supplementary Table
S2) or after stratifying the analysis by use of proton pump inhibitors within 1 year before cancer diagnosis (Supplementary Table S3).

A lower rate of depression, anxiety, and stressrelated disorders, although not statistically significant, was suggested for use of COX-1 selective NSAIDs (HR, 0.93; 95\% CI, 0.85-1.01) compared with no NSAID use (Supplementary Table S4). The use of COX-2 selective NSAIDs was associated with a higher rate (HR, 1.26; 95\% CI, 1.15-1.38) of the studied disorders compared with no NSAID use. A combined use of COX-1 selective, COX-2 selective, and nonselective NSAIDs was associated with a moderately increased rate of the studied disorders.

Table 2 Hazard ratios (95\% confidence intervals) of depression, anxiety, and stress-related disorders during the year after cancer diagnosis, in relation to pre-diagnostic use of NSAIDs

\begin{tabular}{llllll}
\hline Group & 1000 PYs & Event (IR) & Model 1 & Model 2 $^{\text {b }}$ & Model 3 $^{c}$ \\
\hline No NSAIDs & 174 & $3408(19.6)$ & 1.00 & 1.00 & 1.00 \\
Aspirin & 50 & $797(16.1)$ & $0.88(0.80-0.96)$ & $0.86(0.79-0.95)$ & $0.88(0.81-0.97)$ \\
Non-aspirin NSAIDs & 43 & $1145(26.4)$ & $1.26(1.18-1.35)$ & $1.27(1.18-1.36)$ & $1.24(1.15-1.32)$ \\
Both NSAIDs & 13 & $263(20.9)$ & $1.05(0.91-1.20)$ & $1.04(0.91-1.19)$ & $1.05(0.92-1.20)$
\end{tabular}

Cancer patients with any diagnosis of depression, anxiety, or stress-related disorders, namely post-traumatic stress disorder, acute stress reaction, adjustment disorder, or other stress reactions before cancer diagnosis were excluded from the analysis. IRs were calculated by dividing the number of patients that received a diagnosis of depression, anxiety, and stress-related disorders by the number of person-years accumulated during follow-up. Multivariable Cox proportional hazard models were used to estimate the hazard ratios and $95 \%$ confidence intervals in relation to exclusive NSAID use, using no use of NSAIDs as the reference Abbreviations: $P Y$ s person-years, $I R$ incidence rate per 1000 person-years

${ }^{a}$ Estimates were adjusted for age, sex, calendar year of cancer diagnosis, educational level, occupation, region of residence, marital status, and Chronic Disease Score

${ }^{\mathrm{b}}$ Estimates were additionally adjusted for potential indications for NSAID use

'Estimates were additionally adjusted for common cancer types and cancer stage, as well as subtypes of hematological malignancies (leukemia, lymphoma, myeloma, myelodysplastic syndrome, or myeloproliferative neoplasm) 
Recency, daily dose, and duration of aspirin use

The association of aspirin with reduced rate of depression, anxiety, and stress-related disorders was primarily noted among current users (HR, 0.84; $95 \% \mathrm{CI}, 0.75$ to 0.93 ), lowdose users (HR, $0.88 ; 95 \% \mathrm{CI}, 0.80$ to 0.98 ), and long-term users (HR, 0.84; 95\% CI, 0.76 to 0.94) (Table 3). Among commonly combined regimens, individuals with a combination of current, low-dose, and long-term aspirin use had the lowest rate of the studied disorders after cancer diagnosis (HR, 0.77; 95\% CI, 0.68 to 0.87 ).

\section{Subgroup analysis and effect modification}

The magnitude of the inverse association of aspirin use alone with the rate of depression, anxiety, and stressrelated disorders was greater among females than males, among patients who lived in the east of Sweden than patients who lived in other parts of Sweden, among patients with cardiovascular disease than patients without cardiovascular disease, and among breast cancer patients than patients with other cancer types (all $P$ values for interaction < 0.05) (Table 4). The association did not, however, differ by calendar period of cancer diagnosis, age, educational level, marital status, occupation, comorbidity, other indications for NSAID use, or cancer stage. Similar result patterns were found for current use, longterm use, and low-dose use of aspirin.

\section{Discussion}

To the best of our knowledge, this nationwide registerbased cohort study is the first to examine the association of prior NSAID use with the risk of depression, anxiety, and stress-related disorders after cancer diagnosis. The study included all patients with newly diagnosed cancer in Sweden between July 2006 and December 2013 and found that aspirin use, especially long-term and lowdose use, shortly before cancer diagnosis was associated with a reduced rate of depression, anxiety, and stressrelated disorders during the first year after cancer

Table 3 Hazard ratios (95\% confidence intervals) of depression, anxiety, and stress-related disorders during the year after cancer diagnosis in relation to pre-diagnostic exclusive use of aspirin, analysis by recency of use, daily dose, and duration of use

\begin{tabular}{|c|c|c|c|c|c|}
\hline Characteristics & $1000 \mathrm{PYs}$ & Event (IR) & Model $1^{a}$ & Model $2^{b}$ & Model $3^{c}$ \\
\hline No NSAIDs (reference group) & 174 & $3408(19.6)$ & 1.00 & 1.00 & 1.00 \\
\hline \multicolumn{6}{|l|}{ Recency of aspirin use } \\
\hline Previous & 12 & $229(19.1)$ & $1.02(0.89-1.18)$ & $1.02(0.88-1.18)$ & $1.01(0.88-1.17)$ \\
\hline Current & 38 & $568(15.1)$ & $0.82(0.74-0.91)$ & $0.81(0.73-0.90)$ & $0.84(0.75-0.93)$ \\
\hline \multicolumn{6}{|l|}{ Daily dose of aspirin use } \\
\hline Low-dose & 40 & $659(16.4)$ & $0.87(0.79-0.97)$ & $0.87(0.78-0.96)$ & $0.88(0.80-0.98)$ \\
\hline Medium dose & 6 & $90(15.8)$ & $0.95(0.76-1.18)$ & $0.94(0.75-1.17)$ & $0.96(0.77-1.19)$ \\
\hline High dose & 1 & $18(19.9)$ & $1.17(0.73-1.86)$ & $1.16(0.72-1.84)$ & $1.17(0.73-1.86)$ \\
\hline \multicolumn{6}{|l|}{ Duration of aspirin use } \\
\hline Short-term & 10 & $210(20.6)$ & $1.07(0.93-1.25)$ & $1.06(0.92-1.24)$ & $1.07(0.92-1.25)$ \\
\hline Long-term & 37 & $557(15.2)$ & $0.83(0.74-0.92)$ & $0.82(0.74-0.91)$ & $0.84(0.76-0.94)$ \\
\hline \multicolumn{6}{|l|}{ Combined regimens } \\
\hline Previous, low-dose & 10 & 195 (19.3) & $1.03(0.88-1.21)$ & $1.02(0.87-1.19)$ & $1.02(0.87-1.19)$ \\
\hline Previous, medium-high dose & 2 & $30(17.8)$ & $1.09(0.75-1.57)$ & $1.06(0.74-1.53)$ & $1.04(0.72-1.49)$ \\
\hline Current, low-dose, long-term & 26 & $362(14.2)$ & $0.76(0.67-0.86)$ & $0.75(0.66-0.85)$ & $0.77(0.68-0.87)$ \\
\hline Current, low-dose, short-term & 5 & $102(22.1)$ & $1.15(0.94-1.41)$ & $1.13(0.92-1.38)$ & $1.16(0.94-1.42)$ \\
\hline Current, medium-high dose, long-term & 4 & $65(15.5)$ & $0.94(0.73-1.21)$ & $0.92(0.71-1.18)$ & $0.95(0.74-1.23)$ \\
\hline Current, medium-high dose, short-term & 1 & $13(18.1)$ & $1.04(0.60-1.80)$ & $1.02(0.59-1.76)$ & $1.05(0.61-1.82)$ \\
\hline
\end{tabular}

Recency was defined by the time since last dispensed date to cancer diagnosis and patients were classified into previous users (more than 90 days before cancer diagnosis) and current users (within 90 days before cancer diagnosis). A total dispensed dose of aspirin, average daily dose, and number of days to be covered were calculated according to prescription text. Average daily dose was then categorized as low (20-150 mg), medium (151-300 mg), and high (>300 mg) dose. Long-term users were defined as those who used aspirin for $\geq 300$ days during the 365 days before cancer diagnosis. IRs were calculated by dividing the number of patients that received a diagnosis of depression, anxiety, and stress-related disorders by the number of person-years accumulated during follow-up. Combined regimens across recency, dose, and duration were also examined. Hazard ratios and $95 \%$ confidence intervals were estimated from separate Cox proportional hazard models to assess the effect of recency, dose, and duration of use, as well as the six combined regimens of aspirin use, using no use of NSAIDs as the reference

Abbreviations: PYs person-years, IR incidence rate per 1000 person-years

${ }^{a}$ Estimates were adjusted for age, sex, calendar year at cancer diagnosis, educational level, occupation, region of residence, marital status, and Chronic Disease Score

${ }^{b}$ Estimates were additionally adjusted for potential indications for NSAID use

"Estimates were additionally adjusted for common cancer types and cancer stage, as well as subtypes of hematological malignancies (leukemia, lymphoma, myeloma, myelodysplastic syndrome, or myeloproliferative neoplasm) 
Table 4 Hazard ratios (95\% confidence intervals) of depression, anxiety, and stress-related disorders during the year after cancer diagnosis in relation to pre-diagnostic exclusive use of aspirin, stratified analysis by different factors

\begin{tabular}{|c|c|c|c|c|}
\hline Characteristics & Any exclusive aspirin use & Current aspirin & Low-dose aspirin & Long-term aspirin \\
\hline \multicolumn{5}{|l|}{ Calendar year at cancer diagnosis } \\
\hline $2006-2009$ & $0.82(0.71-0.95)$ & $0.74(0.63-0.87)$ & $0.82(0.70-0.97)$ & $0.77(0.65-0.92)$ \\
\hline 2010-2013 & $0.93(0.82-1.06)$ & $0.90(0.78-1.04)$ & $0.93(0.81-1.06)$ & $0.89(0.77-1.03)$ \\
\hline$P$ for interaction & 0.72 & 0.36 & 0.66 & 0.70 \\
\hline \multicolumn{5}{|l|}{ Sex } \\
\hline Male & $0.96(0.83-1.11)$ & $0.92(0.79-1.07)$ & $0.97(0.83-1.12)$ & $0.90(0.77-1.06)$ \\
\hline Female & $0.80(0.70-0.92)$ & $0.74(0.63-0.86)$ & $0.80(0.69-0.93)$ & $0.77(0.65-0.90)$ \\
\hline$P$ for interaction & $<0.01$ & $<0.01$ & $<0.01$ & 0.01 \\
\hline \multicolumn{5}{|l|}{ Age at cancer diagnosis, years } \\
\hline$<50$ & $0.80(0.42-1.54)$ & $0.80(0.38-1.71)$ & $0.86(0.44-1.71)$ & $0.87(0.37-2.07)$ \\
\hline $50-59$ & $0.89(0.65-1.21)$ & $0.79(0.55-1.13)$ & $0.87(0.63-1.20)$ & $0.71(0.48-1.05)$ \\
\hline $60-69$ & $0.89(0.74-1.08)$ & $0.88(0.71-1.08)$ & $0.91(0.75-1.11)$ & $0.84(0.68-1.04)$ \\
\hline$\geq 70$ & $0.90(0.79-1.03)$ & $0.84(0.73-0.97)$ & $0.91(0.79-1.04)$ & $0.89(0.77-1.02)$ \\
\hline$P$ for interaction & 0.82 & 0.92 & 0.93 & 0.32 \\
\hline \multicolumn{5}{|l|}{ Education level } \\
\hline Low & $0.89(0.76-1.03)$ & $0.85(0.72-1.01)$ & $0.89(0.76-1.05)$ & $0.87(0.74-1.03)$ \\
\hline Medium & $1.02(0.87-1.20)$ & $0.93(0.78-1.12)$ & $1.04(0.88-1.23)$ & $0.96(0.80-1.15)$ \\
\hline High & $0.67(0.53-0.84)$ & $0.66(0.51-0.85)$ & $0.63(0.49-0.81)$ & $0.58(0.45-0.77)$ \\
\hline$P$ for interaction & 0.14 & 0.58 & 0.05 & 0.07 \\
\hline \multicolumn{5}{|l|}{ Marital status } \\
\hline Unmarried & $0.72(0.54-0.96)$ & $0.76(0.55-1.04)$ & $0.78(0.58-1.06)$ & $0.72(0.52-1.01)$ \\
\hline Married/registered partnership & $0.86(0.74-0.99)$ & $0.79(0.67-0.93)$ & $0.84(0.72-0.98)$ & $0.78(0.66-0.91)$ \\
\hline Divorced/separated & $0.97(0.78-1.20)$ & $0.96(0.75-1.22)$ & $0.98(0.78-1.23)$ & $0.99(0.77-1.26)$ \\
\hline Widow(er)/surviving partner & $0.97(0.78-1.20)$ & $0.88(0.69-1.11)$ & $0.98(0.78-1.23)$ & $0.95(0.75-1.20)$ \\
\hline$P$ for interaction & 0.82 & 0.78 & 0.76 & 0.27 \\
\hline \multicolumn{5}{|l|}{ Occupation } \\
\hline Blue-collar & $0.89(0.61-1.29)$ & $0.80(0.52-1.23)$ & $0.88(0.60-1.30)$ & $0.86(0.56-1.32)$ \\
\hline White-collar & $0.80(0.58-1.11)$ & $0.74(0.51-1.07)$ & $0.77(0.55-1.09)$ & $0.60(0.40-0.91)$ \\
\hline Not working & $0.89(0.80-1.00)$ & $0.85(0.75-0.95)$ & $0.90(0.81-1.01)$ & $0.87(0.77-0.97)$ \\
\hline$P$ for interaction & 0.93 & 0.92 & 0.86 & 0.84 \\
\hline \multicolumn{5}{|l|}{ Place of residence } \\
\hline East & $0.83(0.71-0.97)$ & $0.76(0.64-0.91)$ & $0.86(0.73-1.01)$ & $0.79(0.67-0.94)$ \\
\hline South & $0.93(0.81-1.08)$ & $0.93(0.79-1.10)$ & $0.93(0.80-1.09)$ & $0.90(0.76-1.06)$ \\
\hline North & $0.87(0.69-1.12)$ & $0.73(0.56-0.97)$ & $0.84(0.65-1.09)$ & $0.80(0.61-1.05)$ \\
\hline$P$ for interaction & $<0.01$ & $<0.01$ & 0.01 & 0.01 \\
\hline \multicolumn{5}{|l|}{ Chronic disease score $^{c}$} \\
\hline $1-3$ & $0.91(0.79-1.04)$ & $0.84(0.72-0.98)$ & $0.91(0.79-1.05)$ & $0.86(0.74-1.01)$ \\
\hline$>3$ & $0.87(0.75-1.00)$ & $0.83(0.71-0.97)$ & $0.88(0.75-1.02)$ & $0.82(0.70-0.96)$ \\
\hline$P$ for interaction & 0.39 & 0.59 & 0.38 & 0.35 \\
\hline \multicolumn{5}{|l|}{ Cardiovascular disease } \\
\hline No & $0.95(0.84-1.07)$ & $0.88(0.76-1.01)$ & $0.97(0.85-1.10)$ & $0.90(0.78-1.03)$ \\
\hline Yes & $0.81(0.68-0.95)$ & $0.79(0.66-0.94)$ & $0.79(0.66-0.93)$ & $0.78(0.66-0.93)$ \\
\hline$P$ for interaction & 0.01 & 0.12 & 0.01 & 0.07 \\
\hline
\end{tabular}


Table 4 Hazard ratios (95\% confidence intervals) of depression, anxiety, and stress-related disorders during the year after cancer diagnosis in relation to pre-diagnostic exclusive use of aspirin, stratified analysis by different factors (Continued)

\begin{tabular}{|c|c|c|c|c|}
\hline Characteristics & Any exclusive aspirin use & Current aspirin & Low-dose aspirin & Long-term aspirin \\
\hline \multicolumn{5}{|c|}{ Inflammatory musculoskeletal condition } \\
\hline No & $0.90(0.81-1.01)$ & $0.84(0.74-0.95)$ & $0.92(0.82-1.03)$ & $0.86(0.76-0.97)$ \\
\hline Yes & $0.82(0.66-1.02)$ & $0.81(0.64-1.03)$ & $0.76(0.60-0.96)$ & $0.78(0.61-0.99)$ \\
\hline$P$ for interaction & 0.49 & 0.90 & 0.16 & 0.54 \\
\hline \multicolumn{5}{|l|}{ Inflammatory systemic disease } \\
\hline No & $0.88(0.80-0.97)$ & $0.83(0.74-0.93)$ & $0.89(0.80-0.98)$ & $0.84(0.75-0.94)$ \\
\hline Yes & $0.93(0.51-1.69)$ & $0.85(0.43-1.67)$ & $0.80(0.41-1.57)$ & $0.75(0.37-1.54)$ \\
\hline$P$ for interaction & 0.88 & 0.85 & 0.47 & 0.58 \\
\hline \multicolumn{5}{|l|}{ Pain and fever } \\
\hline No & $0.88(0.80-0.97)$ & $0.83(0.74-0.92)$ & $0.88(0.79-0.98)$ & $0.84(0.75-0.94)$ \\
\hline Yes & $0.98(0.52-1.84)$ & $1.04(0.52-2.06)$ & $0.92(0.47-1.80)$ & $0.61(0.28-1.36)$ \\
\hline$P$ for interaction & 0.90 & 0.66 & 0.98 & 0.42 \\
\hline \multicolumn{5}{|l|}{ Cancer stage } \\
\hline Localized limited & $0.78(0.63-0.95)$ & $0.71(0.56-0.89)$ & $0.79(0.64-0.97)$ & $0.73(0.58-0.92)$ \\
\hline Localized advanced & $1.06(0.75-1.50)$ & $1.12(0.77-1.64)$ & $0.95(0.65-1.39)$ & $0.82(0.55-1.24)$ \\
\hline Regional spread & $0.78(0.61-0.99)$ & $0.72(0.55-0.95)$ & $0.77(0.60-1.00)$ & $0.73(0.56-0.96)$ \\
\hline Distant metastasis & $1.01(0.76-1.34)$ & $0.98(0.72-1.33)$ & $1.10(0.83-1.47)$ & $1.08(0.80-1.47)$ \\
\hline$P$ for interaction & 0.57 & 0.45 & 0.23 & 0.14 \\
\hline \multicolumn{5}{|l|}{ Cancer type } \\
\hline Prostate cancer & $0.94(0.71-1.26)$ & $0.88(0.64-1.20)$ & $0.93(0.68-1.26)$ & $0.77(0.56-1.07)$ \\
\hline Breast cancer & $0.74(0.56-0.98)$ & $0.63(0.46-0.87)$ & $0.73(0.54-0.97)$ & $0.71(0.52-0.98)$ \\
\hline Gastrointestinal cancer & $1.03(0.84-1.27)$ & $1.05(0.84-1.32)$ & $1.02(0.82-1.27)$ & $1.03(0.82-1.29)$ \\
\hline Lung cancer & $0.78(0.58-1.05)$ & $0.70(0.50-0.97)$ & $0.82(0.61-1.12)$ & $0.73(0.53-1.01)$ \\
\hline Skin cancer excl. Melanoma & $1.07(0.66-1.74)$ & $0.89(0.52-1.53)$ & $1.09(0.65-1.84)$ & $1.19(0.71-1.99)$ \\
\hline Melanoma & $0.56(0.30-1.03)$ & $0.54(0.28-1.05)$ & $0.45(0.23-0.91)$ & $0.42(0.20-0.89)$ \\
\hline Kidney and bladder & $0.86(0.59-1.26)$ & $0.80(0.52-1.23)$ & $0.85(0.56-1.27)$ & $0.92(0.61-1.39)$ \\
\hline Gynecologic cancer & $0.76(0.52-1.12)$ & $0.83(0.55-1.25)$ & $0.78(0.52-1.16)$ & $0.68(0.44-1.05)$ \\
\hline Hematological malignancies & $1.16(0.82-1.66)$ & $1.25(0.85-1.83)$ & $1.25(0.87-1.80)$ & $1.18(0.79-1.76)$ \\
\hline Other cancers & $0.80(0.62-1.05)$ & $0.67(0.49-0.91)$ & $0.83(0.63-1.09)$ & $0.74(0.55-1.01)$ \\
\hline$P$ for interaction & 0.04 & 0.03 & 0.15 & 0.12 \\
\hline
\end{tabular}

The columns refer to four definitions of exposure in separate models: any exclusive use of aspirin, current use of aspirin, low-dose use of aspirin, and long-term use of aspirin, where no use of NSAIDs was used as the reference in all models. Stratum-specific hazard ratios were estimated for each exposure by fitting separate Cox proportional hazard models for different levels of the stratification variable and are presented in different rows, adjusting for age, sex, calendar year at cancer diagnosis, educational level, occupation, region of residence, marital status, Chronic Disease Score, potential indications for NSAIDs, cancer type, cancer stage, and subtypes of hematological malignancies. To assess the interaction between the exposure and each stratification variable, multivariable models were fitted adjusting for the above covariates and including an interaction term for the exposure and each level of the individual stratification variable. P for interaction was then calculated through a Wald test of the null hypothesis that interaction parameter for the exposure and the individual stratification variable is equal to zero. $P<0.05$ indicates a statistically significant interaction between the exposure and the stratification variable

diagnosis. Non-aspirin NSAID use, on the other hand, was associated with a higher rate of depression, anxiety, and stress-related disorders.

The different results for aspirin and non-aspirin NSAI Ds are consistent with recent studies of depression in the general population [25] and among patients with stroke [26]. We further extended the knowledge to other common psychiatric disorders, including anxiety and stress-related disorders (namely PTSD, acute stress reaction, adjustment disorder, and other stress reactions). The different results for aspirin and non-aspirin NSAIDs are also biologically plausible. Accumulating evidence supports the role of COX-1 inhibition in attenuating neuroinflammation, leading to protection against inflammatory brain damage [28, 38, 39]. In contrast, COX-2 inhibitors have been shown to augment nitro-oxidative and oxidative stress in the brain [40], and to interfere with the resolution of inflammation by 
decreasing the negative immunoregulator Prostaglandin E2 [41], thereby aggravate neuroinflammation. Consistent with this, selective COX-2 inhibitors have also been related to increased psychiatric symptoms including depression, anxiety, and changes in cognition [42]. Taken together, COX-1, rather than COX-2 inhibition, might be the key factor in blocking neuroinflammation [38]. Our findings that COX-2 selective NSAID use was associated with a higher risk of common psychiatric disorders compared with nonselective NSAID use also supports this hypothesis. Aspirin selectively inhibits COX-1, particularly at a low dose [38]. As the majority of aspirin is used at low dose, the observed protective effect of aspirin on the studied disorders is likely driven by COX-1 inhibition. The null association of high-dose aspirin may on the other hand be due to additional inhibition of COX-2 [25]. In contrast, although non-aspirin NSAIDs have mixed selectivity, they are mostly selective for COX-2 inhibition or non-selective, possibly leading to the observed harmful effect of non-aspirin NSAIDs on these psychiatric disorders. This was however partly inconsistent with the findings of a recent meta-analysis that found celecoxib to have an antidepressant effect when added to traditional antidepressants [24]. One possible explanation for the contradictory findings might be the fact that we studied newly onset depression, anxiety, and stress-related disorders after cancer diagnosis whereas Bai et al. studied the treatment effort on prevalent depression [24].

We further found that current, long-term, and lowdose aspirin use was associated with the lowest rate of depression, anxiety, and stress-related disorders after cancer diagnosis. Current and long-term aspirin use reflects persistent anti-inflammatory activity, in addition to low-dose use with enhanced inhibition of COX-1. The stratified results by cancer stage showed clear evidence for an inverse association between aspirin and the studied disorders in most stages of cancer, apart from distant metastatic cancer. Furthermore, the effect of aspirin was more pronounced among females, in line with the proposed female-specific impact of low-dose aspirin in antiinflammation [43]. The stronger association noted for females might alternatively be attributable to the stronger findings among breast cancer patients, which corroborates with our preclinical findings using animal models [28]. The underlying reasons for the stronger association among patients with breast cancer, compared with patients with other cancers, remain unknown. This finding, together with the fact that patients with breast cancer have indeed the highest prevalence of depression among patients of different cancer types [33], highlights a potentially specific role of inflammation in breast cancerrelated depression [44].

The strength of the study includes the nationwide population-based study design, independent collection of information on drug exposures and psychiatric disorders, the comprehensive information of covariables, and thorough statistical analysis including multivariable adjustments for potential confounders. Common systemic and random errors are therefore minimized. Some potential limitations of the study should, however, still be noted. The Prescribed Drug Register does not include information on medications used over the counter or in hospitals and nursing homes. We speculate however that this is non-differential between individuals that would later receive a cancer diagnosis and subsequently also a diagnosis of depression, anxiety, and stress-related disorders and individuals that would not receive a diagnosis of psychiatric disorders after being diagnosed with cancer. Such misclassification would therefore most likely have diluted the magnitude of the studied association. Also, this should not have affected the results on aspirin use greatly because low-dose aspirin is mostly prescribed in Sweden [45]. Further, because of its observational nature, residual confounding due to unknown and unmeasured confounders may exist. Patients with a pre-existing psychiatric disorder might be more likely to use NSAIDs and at higher risk of depression, anxiety, and stressrelated disorders after cancer diagnosis. We therefore excluded patients with any psychiatric disorders-in addition to patients with depression, anxiety, and stressrelated disorders-prior to cancer diagnosis and observed similar results. However, the exclusion of these patients was likely incomplete because not all patients with psychiatric disorders attend health care. Similarly, we investigated the role of gastrointestinal symptoms, which could indicate high stress levels and reduced NSAID use, by stratifying the analysis by use of proton pump inhibitors during the year before cancer diagnosis and found again similar results.

\section{Conclusions}

Aspirin use, especially current, long-term, and low-dose use, was associated with a decreased risk of depression, anxiety, and stress-related disorders following cancer diagnosis, while the use of non-aspirin NSAIDs was associated with an increased risk, compared with no use of NSAIDs. Our findings call for pre-clinical research in examining the underlying mechanisms of low-dose aspirin and depression, anxiety, and stress-related disorders after cancer diagnosis and, if confirmed in further studies, provide a rationale for randomized clinical trials.

\section{Supplementary information}

Supplementary information accompanies this paper at https://doi.org/10. 1186/s12916-020-01709-4.

Additional file 1: Table S1. Hazard ratios (95\% confidence intervals) of depression, anxiety, or stress-related disorder during the year after cancer 
diagnosis in relation to pre-diagnostic use of NSAIDs. Table S2. Hazard ratios (95\% confidence intervals) of depression, anxiety, and stress-related disorders during the year after cancer diagnosis in relation to prediagnostic use of NSAIDs, after excluding patients with any psychiatric disorders before cancer diagnosis. Table S3. Hazard ratios (95\% confidence intervals) of depression, anxiety, and stress-related disorders during the year after cancer diagnosis in relation to pre-diagnostic NSAID use, stratified analysis by pre-diagnostic use of proton pump inhibitors (PPIs). Table S4. Hazard ratios (95\% confidence intervals) of depression, anxiety, and stress-related disorders during the year after cancer diagnosis in relation to pre-diagnostic use of NSAIDs, analysis by selectivity of NSAIDs.

\section{Abbreviations}

Cl: Confidence interval; COX: Cyclooxygenase; FIGO: International Federation of Gynecology and Obstetrics; HR: Hazard ratio; ICD-10: International Statistical Classification of Diseases and Related Health Problems 10th Revision; LISA: Longitudinal Integrated Database for Health Insurance and Labour Market Studies; NSAIDs: Non-steroidal anti-inflammatory drugs; PTSD: Post-traumatic stress disorder

\section{Acknowledgements}

We thank Qing Shen from Karolinska Institutet for helping with coding in SAS.

\section{Authors' contributions}

FF, AW, and ES conceived the study idea. $\mathrm{KH}, \mathrm{AS}, \mathrm{DL}$, and FF planned and conducted the analysis. KH and FF drafted the manuscript. All authors interpreted the results, revised the manuscript substantially, and approved the manuscript for publication.

\section{Funding}

This study was supported by grants awarded to FF by Swedish Cancer Society (No. CAN 2017/322) and the Swedish Research Council for Health, Working Life and Welfare (No. 2017-00531), to KH by China Scholarship Council (No. 201806240005), to ES by National Health and Medical Research Council (GNT1147498) and National Breast Cancer Foundation (IIRS-20 to 025), and to AW by the National Breast Cancer Foundation (PF-15 to 014). The researchers were independent of the funding agencies. The funding bodies have no role in the design of the study or collection, analysis, and interpretation of data or in writing the manuscript. Open access funding provided by Karolinska Institute.

\section{Availability of data and materials}

Data are from the Swedish Cancer Register, Causes of Death Register, Migration Register, Patient Register, and Prescribed Drug Register. Data cannot be put into a public data repository due to Swedish law but are available by applying through Statistics Sweden (for Migration Register) and the Swedish National Board of Health and Welfare (for the other four registers). Detailed information on data application is in the following links: https://www.scb.se/vara-tjanster/bestalla-mikrodata/ and https://bestalladata. socialstyrelsen.se/.

\section{Ethics approval and consent to participate}

This study was approved by the Regional Ethics Review Board in Stockholm, Sweden (Dnr 2015/1574-31). No personal consent is needed from the register-based study participants, as the study involves no direct contact with the participants and all data were anonymized and non-identifiable.

\section{Consent for publication}

It was not necessary to gain consent from individual participants for publication since anonymous data in such a way that the individual is no longer identifiable is not considered to be personal data, and thus not governed by the European Union General Data Protection Regulation.

\section{Competing interests}

The authors declare that they have no competing interests.

\section{Author details}

'Unit of Integrative Epidemiology, Institute of Environmental Medicine, Karolinska Institutet, Box 210, 17177 Stockholm, Sweden. ${ }^{2}$ Department of
Medical Epidemiology and Biostatistics, Karolinska Institutet, Stockholm, Sweden. ${ }^{3}$ Channing Division of Network Medicine, Brigham and Women's Hospital, Harvard Medical School, Boston, MA, USA. ${ }^{4}$ Department of Epidemiology, Harvard T.H. Chan School of Public Health, Boston, MA, USA. ${ }^{5}$ Drug Discovery Biology Theme, Monash Institute of Pharmaceutical Sciences, Monash University, Parkville, Victoria 3052, Australia. ${ }^{6}$ Laboratory of ImmunoPsychiatry, Neuroscience Research Australia, Randwick, New South Wales 2031, Australia. 'School of Psychiatry, University of New South Wales, Sydney 2052, Australia. ${ }^{8}$ Clinical Epidemiology and Biostatistics School of Medical Sciences, Örebro Universitet, Örebro, Sweden. ${ }^{9}$ Centre of Public Health Sciences Faculty of Medicine, University of Iceland, Reykjavík, Iceland.

${ }^{10}$ Department of Oncology, Södersjukhuset, Stockholm, Sweden. "1'Division of Clinical Epidemiology, Department of Medicine Solna, Karolinska Institutet, Stockholm, Sweden.

Received: 28 April 2020 Accepted: 14 July 2020

Published online: 09 September 2020

\section{References}

1. Mitchell AJ, Chan M, Bhatti H, Halton M, Grassi L, Johansen C, et al. Prevalence of depression, anxiety, and adjustment disorder in oncological, haematological, and palliative-care settings: a meta-analysis of 94 interviewbased studies. Lancet Oncol. 2011;12(2):160-74.

2. Zhu J, Sjölander A, Fall K, Valdimarsdottir U, Fang F. Mental disorders around cancer diagnosis and increased hospital admission rate - a nationwide cohort study of Swedish cancer patients. BMC Cancer. 2018; 18(1):322

3. Zhu J, Fang F, Sjölander A, Fall K, Adami HO, Valdimarsdóttir U. First-onset mental disorders after cancer diagnosis and cancer-specific mortality: a nationwide cohort study. Ann Oncol. 2017;28(8):1964-9.

4. Batty GD, Russ TC, Stamatakis E, Kivimäki M. Psychological distress in relation to site specific cancer mortality: pooling of unpublished data from 16 prospective cohort studies. BMJ. 2017;356:j108.

5. Lu D, Andersson TML, Fall K, Hultman CM, Czene K, Valdimarsdóttir U, et al. Clinical diagnosis of mental disorders immediately before and after cancer diagnosis: a nationwide matched cohort study in Sweden. JAMA Oncol. 2016;2(9):1188-96.

6. Schumacher JR, Palta M, Loconte NK, Trentham-Dietz A, Witt WP, Heidrich SM, et al. Characterizing the psychological distress response before and after a cancer diagnosis. J Behav Med. 2013;36(6):591-600.

7. Kim GM, Kim SJ, Song SK, Kim HR, Kang BD, Noh SH, et al. Prevalence and prognostic implications of psychological distress in patients with gastric cancer. BMC Cancer. 2017;17(1):283.

8. McCaffery KJ, Irwig L, Turner R, Chan SF, Macaskill P, Lewicka M, et al. Psychosocial outcomes of three triage methods for the management of borderline abnormal cervical smears: an open randomised trial. BMJ. 2010; 340(7744):460

9. Janelsins MC, Heckler CE, Peppone LJ, Kamen C, Mustian KM, Mohile SG, et al. Cognitive complaints in survivors of breast cancer after chemotherapy compared with age-matched controls: an analysis from a nationwide, multicenter, prospective longitudinal study. J Clin Oncol. 2017;35(5):506-14.

10. Krull KR, Hardy KK, Kahalley LS, Schuitema I, Kesler SR. Neurocognitive outcomes and interventions in long-term survivors of childhood cancer. J Clin Oncol. 2018;36(21):2181-9.

11. Jeantieu M, Gaillat F, Antonini F, Azoulay E, Martin C, Thomas P, et al. Postoperative pain and subsequent ptsd-related symptoms in patients undergoing lung resection for suspected cancer. J Thorac Oncol. 2014;9(3): 362-9.

12. Kim T-H, Rowat AC, Sloan EK. Neural regulation of cancer: from mechanobiology to inflammation. Clin Transl Immunol. 2016;5(5):e78.

13. Greten FR, Grivennikov SI. Inflammation and cancer: triggers, mechanisms, and consequences. Immunity. 2019;51(1):27-41.

14. Coussens LM, Werb Z. Inflammation and cancer. Nature. 2002:420(6917): 860-7.

15. Diakos $\mathrm{Cl}$, Charles KA, McMillan DC, Clarke SJ. Cancer-related inflammation and treatment effectiveness. Lancet Oncol. 2014;15(11):e493-503.

16. Dantzer R, O'Connor JC, Freund GG, Johnson RW, Kelley KW. From inflammation to sickness and depression: when the immune system subjugates the brain. Nat Rev Neurosci. 2008;9(1):46-56.

17. Miller AH. Beyond depression: the expanding role of inflammation in psychiatric disorders. World Psychiatry. 2020;19(1):108-9. 
18. Miller $\mathrm{AH}$, Haroon $\mathrm{E}$, Raison $\mathrm{CL}$, Felger JC. Cytokine targets in the brain: impact on neurotransmitters and neurocircuits. Depress Anxiety. 2013;30(4): 297-306.

19. Michopoulos V, Powers A, Gillespie CF, Ressler KJ, Jovanovic T. Inflammation in fear-and anxiety-based disorders: PTSD, GAD, and beyond. Neuropsychopharmacology. 2017:42(1):254-70.

20. Pervanidou P, Kolaitis G, Charitaki S, Margeli A, Ferentinos S, Bakoula C, et al. Elevated morning serum interleukin (IL)-6 or evening salivary cortisol concentrations predict posttraumatic stress disorder in children and adolescents six months after a motor vehicle accident. Psychoneuroendocrinology. 2007;32(8-10):991-9.

21. Dregan A, Matcham F, Harber-Aschan L, Rayner L, Brailean A, Davis K, et al. Common mental disorders within chronic inflammatory disorders: a primary care database prospective investigation. Ann Rheum Dis. 2019;78(5):688-95.

22. Oates JA, Wood AJJ, Brooks PM, Day RO. Nonsteroidal antiinflammatory drugs — differences and similarities. N Engl J Med. 1991;324(24):1716-25.

23. Meek IL, van de Laar MAFJ, Vonkeman HE. Non-steroidal anti-inflammatory drugs: an overview of cardiovascular risks. Pharmaceuticals. 2010;3(7):214662.

24. Bai S, Guo W, Feng Y, Deng H, Li G, Nie H, et al. Efficacy and safety of antiinflammatory agents for the treatment of major depressive disorder: a systematic review and meta-analysis of randomised controlled trials. J Neurol Neurosurg Psychiatry. 2020;91(1):21-32.

25. Kessing LV, Rytgaard HC, Gerds TA, Berk M, Ekstrøm CT, Andersen PK. New drug candidates for depression - a nationwide population-based study. Acta Psychiatr Scand. 2019;139(1):68-77.

26. Wium-Andersen IK, Wium-Andersen MK, Jørgensen MB, Osler M. Antiinflammatory treatment and risk for depression after first-time stroke in a cohort of 147487 Danish patients. J Psychiatry Neurosci. 2017;42(5):320-30.

27. lyengar RL, Gandhi S, Aneja A, Thorpe K, Razzouk L, Greenberg J, et al. NSAI Ds are associated with lower depression scores in patients with osteoarthritis. Am J Med. 2013;126(11):1017.e11-8.

28. Walker AK, Chang A, Ziegler Al, Dhillon HM, Vardy JL, Sloan EK. Low dose aspirin blocks breast cancer-induced cognitive impairment in mice. PLoS One. 2018;13(12):e0208593.

29. Barlow L, Westergren $K$, Holmberg L, Talbäck M. The completeness of the Swedish Cancer Register - a sample survey for year 1998. Acta Oncol (Madr). 2009;48(1):27-33.

30. Ludvigsson JF, Andersson E, Ekbom A, Feychting M, Kim JL, Reuterwall C, et al. External review and validation of the Swedish national inpatient register. BMC Public Health. 2011;11:450.

31. Wettermark B, Hammar N, MichaelFored C, Leimanis A, Otterblad Olausson $P$, Bergman U, et al. The new Swedish Prescribed Drug Register-opportunities for pharmacoepidemiological research and experience from the first six months. Pharmacoepidemiol Drug Saf. 2007; 16(7):726-35.

32. Mehnert $A$, Brähler $E$, Faller $H$, Härter $M$, Keller $M$, Schulz $H$, et al. Four-week prevalence of mental disorders in patients with cancer across major tumor entities. J Clin Oncol. 2014:32(31):3540-6.

33. Kuhnt S, Brähler E, Faller H, Härter M, Keller M, Schulz H, et al. Twelve-month and lifetime prevalence of mental disorders in cancer patients. Psychother Psychosom. 2016;85(5):289-96.

34. Felger JC. Imaging the role of inflammation in mood and anxiety-related disorders. Curr Neuropharmacol. 2018;16(5):533-88.

35. Lix L, Ps MS, Pitz M, Rashid Ahmed F, Quon H, Griffith J, et al. Cancer data linkage in manitoba: expanding the infrastructure for research. Winnipeg: MB Manitoba Centre for Health Policy; 2016. p. 73-4.

36. Von Korff M, Wagner EH, Saunders K. A chronic disease score from automated pharmacy data. J Clin Epidemiol. 1992;45(2):197-203.

37. Ludvigsson JF, Svedberg P, Olén O, Bruze G, Neovius M. The longitudinal integrated database for health insurance and labour market studies (LISA) and its use in medical research. Eur J Epidemiol. 2019;34(4):423-37.

38. Berk M, Dean O, Drexhage H, McNeil JJ, Moylan S, O'Neil A, et al. Aspirin: a review of its neurobiological properties and therapeutic potential for mental illness. BMC Med. 2013;11(1):74

39. Choi SH, Langenbach R, Bosetti F. Genetic deletion or pharmacological inhibition of cyclooxygenase-1 attenuate lipopolysaccharide-induced inflammatory response and brain injury. FASEB J. 2008;22(5):1491-501.

40. Aid S, Langenbach R, Bosetti F. Neuroinflammatory response to lipopolysaccharide is exacerbated in mice genetically deficient in cyclooxygenase-2. J Neuroinflammation. 2008;5(1):17.
41. Chan MM-Y, Moore AR. Resolution of inflammation in murine autoimmune arthritis is disrupted by cyclooxygenase- 2 inhibition and restored by prostaglandin E2-mediated lipoxin A4 production. J Immunol. 2010;184(11): 6418-26.

42. Onder G, Pellicciotti F, Gambassi G, Bernabei R. NSAID-related psychiatric adverse events. Drugs. 2004;64(23):2619-27.

43. Chiang N, Hurwitz S, Ridker PM, Serhan CN. Aspirin has a gender-dependent impact on antiinflammatory 15-epi-lipoxin A4 formation: a randomized human trial. Arterioscler Thromb Vasc Biol. 2006;26(2):e14-7.

44. Bower JE, Ganz PA, Irwin MR, Kwan L, Breen EC, Cole SW. Inflammation and behavioral symptoms after breast cancer treatment: do fatigue, depression, and sleep disturbance share a common underlying mechanism? J Clin Oncol. 2011;29(26):3517-22.

45. Frisk G, Ekberg S, Lidbrink E, Eloranta S, Sund M, Fredriksson I, et al. No association between low-dose aspirin use and breast cancer outcomes overall: a Swedish population-based study. Breast Cancer Res. 2018;20(1): 142.

\section{Publisher's Note}

Springer Nature remains neutral with regard to jurisdictional claims in published maps and institutional affiliations.
Ready to submit your research? Choose BMC and benefit from:

- fast, convenient online submission

- thorough peer review by experienced researchers in your field

- rapid publication on acceptance

- support for research data, including large and complex data types

- gold Open Access which fosters wider collaboration and increased citations

- maximum visibility for your research: over $100 \mathrm{M}$ website views per year

At BMC, research is always in progress.

Learn more biomedcentral.com/submissions 